\title{
Beneficial impact of intensified multifactorial intervention on risk of stroke: outcome of 21 years of follow-up in the randomised Steno-2 Study
}

\author{
Peter Gæde $^{1,2}$ (D) Jens Oellgaard ${ }^{3} \cdot$ Christina Kruuse $^{4} \cdot$ Peter Rossing $^{5,6} \cdot$ Hans-Henrik Parving $^{7} \cdot$ Oluf Pedersen $^{8}$
}

Received: 29 January 2019 / Accepted: 3 May 2019 / Published online: 1 June 2019

(C) The Author(s) 2019

\begin{abstract}
Aims/hypothesis Epidemiological studies have shown that diabetes is a well-established independent but modifiable risk factor for stroke. The aim of this post hoc analysis of data from the Steno-2 Study was to examine whether multiple risk factor intervention reduced the risk for stroke in individuals with type 2 diabetes and microalbuminuria.

Methods In the Steno-2 Study, 160 individuals with type 2 diabetes and microalbuminuria were randomised to intensified or conventional multiple risk factor intervention, targeting classical cardiovascular disease risk factors for a mean of 7.8 years, and then followed for a total mean of 21.2 years. The primary endpoint in this post hoc analysis was time to first stroke event.

Results During follow-up, 30 participants experienced a total of 39 strokes. Individuals randomised to conventional therapy were more likely to experience a stroke than those in the intensive-therapy group, with 29 total strokes occurring in 21 participants $(26 \%)$ in the conventional-therapy group vs a total of ten strokes in nine participants (11\%) in the intensive-therapy group (HR 0.31 [95\% CI 0.14, 0.69]; $p=0.004$ ). Also, the number of recurrent strokes was significantly reduced with intensive therapy.

Conclusions/interpretation Intensified multiple risk factor intervention in patients with type 2 diabetes and microalbuminuria reduces the risk for strokes as well as the number of recurrent cerebrovascular events.
\end{abstract}

Trial registration ClinicalTrials.gov NCT00320008.

Keywords Microalbuminuria $\cdot$ Multifactorial intervention $\cdot$ Stroke $\cdot$ Type 2 diabetes

Electronic supplementary material The online version of this article (https://doi.org/10.1007/s00125-019-4920-3) contains peer-reviewed but unedited supplementary material, which is available to authorised users.

Oluf Pedersen

oluf@sund.ku.dk

1 Department of Cardiology and Endocrinology, Slagelse Hospital, Slagelse, Denmark

2 Institute for Regional Health Research, University of Southern Denmark, Odense, Denmark

3 Novo Nordisk Scandinavia A/B, Region Denmark, Ørestad, Denmark

4 Department of Neurology, Herlev Gentofte Hospital, University of Copenhagen, Herlev, Denmark
5 Steno Diabetes Center Copenhagen, Gentofte, Denmark

6 Faculty of Health Sciences, University of Copenhagen, Copenhagen, Denmark

7 Department of Endocrinology, Rigshospitalet, Copenhagen, Denmark

Novo Nordisk Foundation Center for Basic Metabolic Research, Section of Metabolic Genetics, Faculty of Health and Medical Sciences, University of Copenhagen, Panum, Maersk Tower, Blegdamsvej 3B, 2200 Copenhagen N, Denmark 


\section{Research in context}

\section{What is already known about this subject?}

- The global lifetime risk for having a stroke in the total population is estimated to be $24.9 \%$

- Stroke events are more common in individuals with diabetes compared with the general population

- Although the risk for stroke in diabetes has diminished, the prevalence is increasing due to an increase in the number of individuals with type 2 diabetes worldwide

\section{What is the key question?}

- Does intensified multiple risk factor intervention reduce the risk for stroke in individuals with type 2 diabetes and microalbuminuria?

\section{What are the new findings?}

- Intensified multiple risk factor intervention reduced the hazard for stroke by $69 \%$

- The risk for recurrent stroke is also significantly reduced by intensified intervention

\section{How might this impact on clinical practice in the foreseeable future?}

- These findings emphasise the importance of following guidelines recommending intensified risk factor control in patients with type 2 diabetes

\author{
Abbreviations \\ CVD Cardiovascular disease \\ TIA Transient ischaemic attacks
}

\section{Introduction}

A recent publication from the Global Burden of Disease (GBD) Study 2016 estimates the global lifetime risk of stroke to be $24.9 \%$ in the total population for both sexes, with an increase in risk of $15.4 \%$ for men and $3.2 \%$ for women compared with the previous survey, which was conducted in 1990 [1].

In individuals with diabetes in the USA, an increased incidence of stroke from 1990 to 2010 has been demonstrated. Although a $52.7 \%$ decrease in the age-standardised rates of stroke was seen, the increase in diabetes prevalence resulted in a $47 \%$ increase in the yearly number of strokes [2].

The importance of individualised intensified multifactorial intervention in the treatment of type 2 diabetes has been highlighted in the Steno-2 Study. In this study, an increased median lifespan of 7.9 years with significant risk reductions for major cardiovascular disease (CVD) events, heart failure and microvascular complications was observed with intensified treatment of multiple risk factors vs conventional therapy [3]. Also, it has been demonstrated that these results were achieved at no extra cost; the total direct cost of the intensified multifactorial intervention was similar to that of conventional treatment [4].

In the present post hoc analysis, the primary aim was to examine the difference in time to first stroke event between the intensive and conventional treatment groups of the Steno-2 Study.

\section{Methods}

Study design The Steno-2 Study (ClinicalTrials.gov registration no. NCT00320008) has been described in detail previously [3-5]. In short, during 1992 and 1993, 160 participants with type 2 diabetes and microalbuminuria were randomised to conventional or intensified multifactorial treatment targeting several concomitant risk factors $(n=80$ in each group; Fig. 1). Mean treatment duration was 7.8 years. After 7.8 years, the randomised part of the study ended and all individuals were offered intensified multifactorial treatment, allowing the trial to continue as an observational follow-up study for an additional 13.4 years.

The protocol for the follow-up trial in the Steno-2 Study was conducted in accordance with the declaration of Helsinki and approved by a local ethics committee (Ethics committee, Capital Region of Denmark; protocol ID number: H-KA99035-GS, add. 41104) and by the Danish Data Protection Agency (J.Nr. 2015-41-4042). All patients gave their informed consent upon randomisation and confirmed this on follow-up visits.

Endpoint definitions All CVD endpoints in the Steno-2 Study were defined a priory and have been described in detail elsewhere [5]. The definitions for stroke and transient ischaemic 
Fig. 1 CONSORT diagram showing participant flow throughout the Steno-2 trial. The first 7.8 years were the active intervention period, after which time the randomisation was neutralised and continued as a post-trial observational follow-up study with all remaining participants being offered the same treatment as the original intensive-therapy group. At the time of randomisation, the mean age was 55.1 years and $66 \%$ were male. All participants had microalbuminuria. Reproduced from [8] under the terms of the Creative Commons Attribution 4.0 International License (http:// creativecommons.org/licenses/ by/4.0/), which permits unrestricted use, distribution and reproduction in any medium

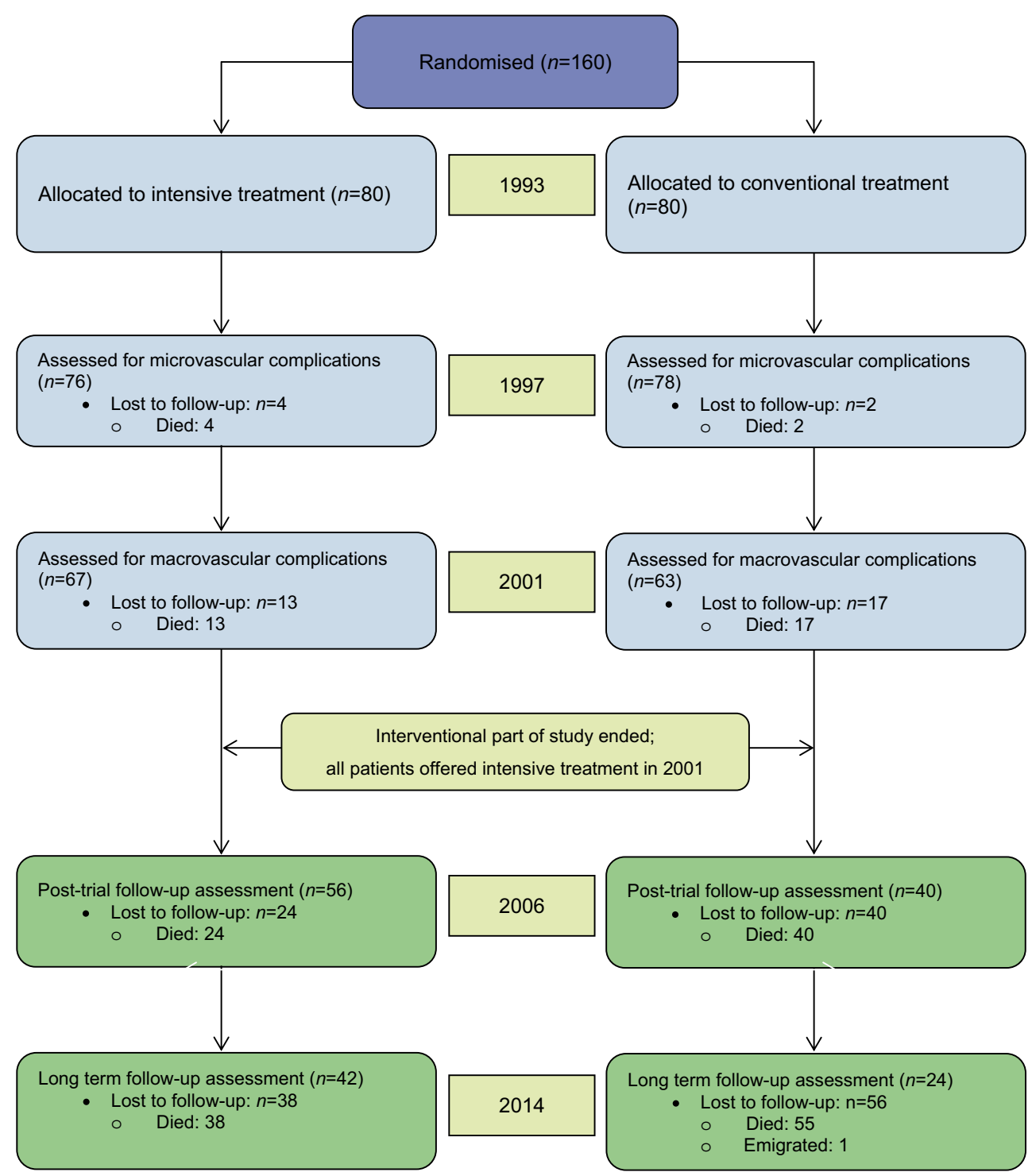

attack (TIA) are shown in the electronic supplementary materials (ESM) Methods. All possible cases of stroke and TIA were adjudicated by an external committee masked for original treatment allocation.

In the current analysis, we distinguish between stroke and TIA. This was not the case in the original Steno-2 Study where we used a combination of the two conditions in order to increase power due to the shorter duration of follow-up and, thus, a smaller number of expected events.

The primary analysis of the present study was time to first stroke event, with the secondary analyses being time to a combined endpoint of stroke and cardiovascular death and time to a combined endpoint of stroke and all-cause mortality. In sensitivity analyses, cases of TIA were added to the endpoints specified above.

Statistical analyses HRs for the primary and secondary outcomes of this post hoc analysis were calculated using Cox regression. Recurrent strokes were compared using Fisher's exact test. Statistical analyses were performed using Stata/IC version 15 (StataCorp, College Station, TX, USA). For the primary outcome, proportional hazards assumption was checked by the 'estat phtest' function of Stata.

\section{Results}

Baseline values in each of the two original treatment groups are shown in the ESM Table 1. During a follow-up of 21.2 years, 30 individuals experienced a total of 39 stroke events. Participants randomised to conventional therapy were more likely to experience a stroke, with a total of 29 strokes occurring in 21 individuals (26\%) in the conventional-therapy group, compared with a total of ten stroke events in nine individuals $(11 \%)$ in the intensive-therapy group. 


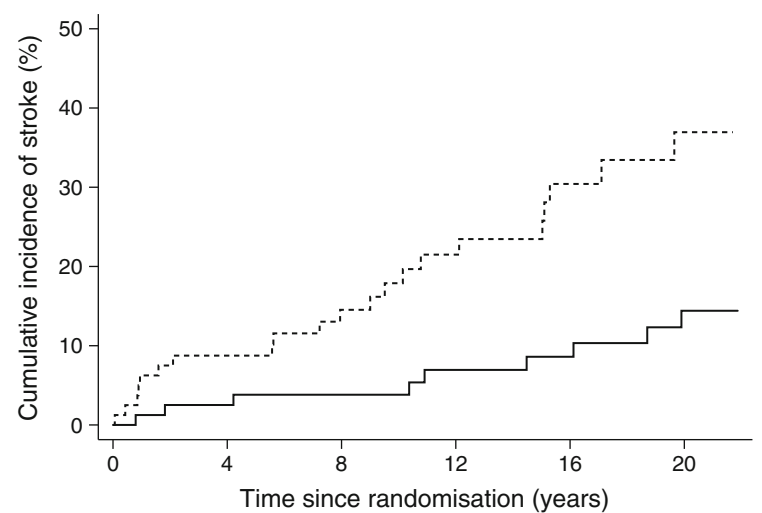

Number at risk

$\begin{array}{llllll}\text { Intensive } & 80 & 75 & 66 & 57 & 53\end{array}$

$\begin{array}{lllllll}\text { Conventional } & 80 & 71 & 57 & 40 & 28 & 18\end{array}$

Fig. 2 Cumulative incidence frequency plot of time to first stroke. The hazard for stroke was reduced by $69 \%$ in the intensive-therapy group $(p=0.004)$. Solid line, intensive-therapy group; dashed line, conventional-therapy group

The hazard for stroke was reduced by $69 \%$ with intensive therapy vs conventional treatment (HR 0.31 [95\% CI 0.14, 0.69]; $p=0.004$; Fig. 2). The pattern was similar for TIA and for the combined event of stroke and TIA (Table 1).

We also demonstrated that intensive therapy resulted in a significantly reduced risk of the combined endpoint of stroke and CVD mortality $(n=55$; HR 0.36 [95\% CI $0.20,0.63$; $p<0.001)$ and for the combined endpoint of stroke and allcause mortality $(n=101$; HR 0.46 [95\% CI $0.31,0.69$; $p<0.001$ ) (Table 1). The median time to stroke or death was 19.9 years in the intensive-therapy group and 11.4 years in the conventional-therapy group.

In the intensive-therapy group, $4(11 \%)$ out of the 35 participants with a cardiovascular event experienced a stroke as their first manifestation of CVD, whereas this was the case in 14 out of 51 individuals $(27 \%)$ in the conventional-therapy group.

Of the total 39 strokes evaluated in the study, 35 were ischaemic. The remaining four were haemorrhagic strokes, two of which were the first stroke in one patient in each of the two treatment groups, with the last two being a recurrent stroke occurring in two different individuals in the conventionally treated group.
Individuals who were originally randomised to conventional treatment were more likely to experience recurrent strokes than those originally randomised to intensified therapy, with one participant in the intensive-therapy group vs ten participants in the conventional-therapy group experiencing more than one stroke $(p=0.018)$.

A similar proportion of individuals in the two groups who experienced a stroke died during follow-up (78\% in the conventional-therapy group vs $71 \%$ in the intensive-therapy group). However, participants in the original intensive-therapy group survived for a significantly shorter period after a stroke than those originally randomised to conventional therapy (median 0.3 years vs 3.1 years, respectively). This pattern was not observed for cardiovascular events not related to strokes (myocardial infarctions, amputations and peripheral or cardiac revascularisation), for which a smaller proportion of individuals randomised to intensive treatment vs conventional treatment died during follow-up after an event (55\% vs $82 \%$ ), with no difference in survival time after the evident event (median 3.4 years vs 3.9 years).

\section{Discussion}

In the present post hoc analysis of data from individuals with type 2 diabetes and microalbuminuria from the Steno- 2 Study with 21.2 years of follow-up, the hazard for first stroke after randomisation was reduced by $69 \%$ and the absolute risk by $15 \%$ with intensive therapy vs conventional treatment. Similarly, individuals originally randomised to conventional treatment were more likely to have recurrent strokes than those originally randomised to intensified therapy. Our findings once again emphasise the importance of an intensified multifaceted intervention approach, according to present EASD/ADA guidelines, for reducing CVD in patients with type 2 diabetes.

The proportion of individuals with a stroke during the follow-up period was $11 \%$ and $26 \%$ in the intensive- and conventional-therapy group, respectively. Since all participants in the Steno-2 Study had microalbuminuria (a marker of general vascular damage, indicating a higher risk for future
Table 1 Number of participants (referring to first event) and HR with $95 \%$ CI of primary and secondary outcomes

\begin{tabular}{lllll}
\hline Outcome & Intensive therapy $(n)$ & Conventional therapy $(n)$ & HR $(95 \%$ CI $)$ & $p$ value \\
\hline Stroke & 9 & 21 & $0.31(0.14,0.69)$ & 0.004 \\
TIA & 1 & 9 & $0.08(0.01,0.66)$ & 0.019 \\
Stroke or TIA & 10 & 25 & $0.28(0.13,0.58)$ & $<0.001$ \\
Stroke + CVD death & 18 & 37 & $0.36(0.20,0.63)$ & $<0.001$ \\
Stroke + all-cause death & 40 & 61 & $0.46(0.31,0.69)$ & $<0.001$ \\
$>1$ stroke & 1 & 10 & - & $0.018^{\text {a }}$ \\
\hline
\end{tabular}

${ }^{a} p$ value calculated using Fisher's exact test 
complications) at baseline, the high incidence of stroke emphasises the importance of early screening for vascular risk factors in individuals with type 2 diabetes.

A surprising finding in the present post hoc analysis was the significantly shorter median lifespan after the first stroke of 0.3 years in the intensive-therapy group vs 3.1 years in the conventional-therapy group. We have no conclusive explanation for this finding. However, since the treatment algorithm for intensive therapy recommended a daily intake of $150 \mathrm{mg}$ acetylsalicylic acid (ASA), it is reassuring that there was no increase in the number of cerebral haemorrhage events in those originally assigned to the intensive-treatment group.

In diabetic nephropathy, the classic Kimmelstiel-Wilson lesions are nodules of hyaline material in the glomerulus that occur due to an increase in mesangial matrix deposition [6]. In the brain, it has been demonstrated that the small arteries and arterioles that supply the territory of lacunar infarcts show segmental arterial disorganisation, fibrinoid degeneration and lipohyalinosis in those with diabetes or hypertension [7]. We have previously shown marked risk reductions for smallvessel disease in terms of progression of diabetic nephropathy with the intensified treatment approach used in the Steno-2 Study $[3,5]$. It could, therefore, be hypothesised that this approach mainly prevents smaller lacunar infarctions associated with cerebral small-vessel disease, while major thromboembolic strokes with more severe symptoms and a poorer prognosis are prevented to a lesser extent.

As previously mentioned, it has been reported that the risk for stroke in individuals with type 2 diabetes has declined on a national scale [2]. However, despite intensified treatment as per the protocol in the Steno-2 Study, a residual risk for stroke of more than $10 \%$ over the duration of the $\sim 20$ year follow-up was observed. Also, due to the increasing number of individuals diagnosed with diabetes and the increased lifespan of these patients, stroke should still be considered a major personal and societal burden.

Strengths and limitations The major strength in the Steno-2 Study is the completeness of data for individuals enrolled in the trial. Except for one individual who emigrated from Denmark, leading to a lack of data during the observational part of the trial, a complete track of all endpoints and hospital admissions was available from Danish National Registers and applied in all analyses.

One major limitation of the study is the small sample size of 160 individuals. Another limitation is that a high-risk group (individuals with type 2 diabetes and microalbuminuria) were selected as the study population. Therefore, the magnitude of risk reduction we demonstrate here might not be attributable to a lower risk population. However, the risk reductions with intensive therapy presented in the current post hoc analysis are in coherence with previous cardiovascular risk reductions reported in the trial, both during its interventional and observational phase. Furthermore, since all participants were offered intensive treatment after the randomised phase, the effects presented may actually be an underestimation of the true effects.

\section{Conclusion}

Our study demonstrates that stroke is a frequent and fatal complication in individuals with type 2 diabetes and microalbuminuria. An intensified multifactorial intervention significantly reduced the occurrence of this outcome, as well as the number of recurrent cerebrovascular events.

Acknowledgements We wish to thank all the participating patients and their families for their willingness to participate and for sharing laughs and sadness during the entire follow-up period. We also wish to thank P. Hildebrandt (Frederiksberg Heart Clinic, Copenhagen, Denmark) and J. Faber (Herlev Hospital, Copenhagen, Denmark) for serving in the endpoint assessment committee throughout follow-up.

Data availability Data are available upon reasonable request to the corresponding author.

Funding The Steno-2 Study, including the 21.2 years of follow-up, was supported by unrestricted grants from Novo Nordisk A/S, Bagsværd, Denmark. Novo Nordisk A/S were not in any way involved in study design or in the collection, analysis or interpretation of data and the present post hoc analysis did not receive any additional funding. The study sponsor was not involved in the design of the study; the collection, analysis, and interpretation of data; writing the report; or the decision to submit the report for publication.

Duality of interest Since completion of data acquisition, data management and data interpretation for the Steno-2 Study's 21.2 years follow-up, JO has been employed by Novo Nordisk Scandinavia A/B, Region Denmark. PR reports having given lectures for AstraZeneca, Bayer and Boehringer Ingelheim, has served as a consultant for AbbVie, AstraZeneca, Bayer, Eli Lilly, Boehringer Ingelheim, Astellas, Janssen and Novo Nordisk (all fees given to the Steno Diabetes Center) and has equity interest in Novo Nordisk. H-HP has equity interest in Merck and receives honoraria from $\mathrm{AbbVie}$ and Novartis. OP has equity interest in Novo Nordisk A/S. The Novo Nordisk Foundation Center for Basic Metabolic Research is an independent research centre at the University of Copenhagen partially funded by an unrestricted donation from the Novo Nordisk Foundation. CK has equity interest in Novo Nordisk A/S. PG declares no duality of interest associated with this manuscript.

Contribution statement OP conceived and designed the original Steno-2 Study, devised and supervised the 21.2 years follow-up, acquired all funding throughout the study and provided key content to the manuscript. $\mathrm{H}-\mathrm{HP}$ contributed to the conception and supervision of the original Steno2 Study and provided key content in interpreting data in the current analysis. PG acquired all data up to and including the 13.3 years examination, handled patient care during the intervention period, planned the 21.2 years follow-up and supervised data acquisition and processing in the present follow-up. JO coordinated and performed the 21.2 years follow-up, acquired all data for the 21.2 years follow-up, supervised laboratory work and patient evaluations, processed data and performed statistical work. PR supervised and facilitated all work performed at Steno Diabetes Center (i.e. the patient assessments for the long-term followup), was responsible for quality assurance/quality control of the 
performed measures, provided key intellectual content for the discussion section and critically reviewed the manuscript. CK provided key intellectual content in interpreting data and critically reviewed the manuscript. The manuscript was drafted by PG with contributions from all other authors. All authors gave final approval for the paper to be published. OP, H-HP and PG are guarantors of this work.

Open Access This article is distributed under the terms of the Creative Commons Attribution 4.0 International License (http:// creativecommons.org/licenses/by/4.0/), which permits unrestricted use, distribution, and reproduction in any medium, provided you give appropriate credit to the original author(s) and the source, provide a link to the Creative Commons license, and indicate if changes were made.

\section{References}

1. The GBD 2016 Lifetime Risk of Stroke Collaborators (2018) Global, regional, and country-specific lifetime risks of stroke, 1990 and 2016. N Engl J Med 379(25):2429-2437. https://doi.org/10.1056/ NEJMoa1 804492

2. Gregg EW, Li Y, Wang J et al (2014) Changes in diabetes-related complications in the United States, 1990-2010. N Engl J Med 370(16):1514-1523. https://doi.org/10.1056/NEJMoa1310799
3. Gæde P, Oellgaard J, Carstensen B et al (2016) Years of life gained by multifactorial intervention in patients with type 2 diabetes mellitus and microalbuminuria: 21 years follow-up on the Steno-2 randomised trial. Diabetologia 59(11):2298-2307. https://doi.org/ 10.1007/s00125-016-4065-6

4. Gæde J, Oellgaard J, Ibsen R et al (2019) A cost analysis of intensified vs conventional multifactorial therapy in individuals with type 2 diabetes: a post hoc analysis of the Steno-2 study. Diabetologia 62(1):147-155. https://doi.org/10.1007/s00125-018-4739-3

5. Gæde PH (2006) Intensified multifactorial intervention in patients with type 2 diabetes and microalbuminuria: rationale and effect on late-diabetic complications. Dan Med Bull 53(3):258-284

6. Kimmelstiel P, Wilson C (1936) Intercapillary lesions in the glomeruli of the kidney. Am J Pathol 12(1):83-98.7

7. Caplan LR (2015) Lacunar infarction and small vessel disease: pathology and pathophysiology. J Stroke 17(1):2-6. https://doi.org/10. 5853/jos.2015.17.1.2

8. Oellgaard J, Gæde P, Rossing P et al (2018) Reduced risk of heart failure with intensified multifactorial intervention in individuals with type 2 diabetes and microalbuminuria: 21 years of follow-up in the randomised Steno-2 study. Diabetologia 61(8):1724-1733. https:// doi.org/10.1007/s00125-018-4642-y

Publisher's note Springer Nature remains neutral with regard to jurisdictional claims in published maps and institutional affiliations. 